\title{
REVIEW
}

\section{The Influence of Stigma on People with Mental Illness}

\section{Minhang $\mathbf{L i}^{*}$}

University of Sydney, Sydney, Haymarket 2007 NSW, Australia

\section{ARTICLE INFO}

Article history

Received: 16 September 2020

Accepted: 18 September 2020

Published Online: 30 October 2020

\section{Keywords:}

Mental illness stigma

Misunderstanding

Stereotype

Mental health

Stigmatized groups

\begin{abstract}
People with mental illness have not only struggled with the psychological and physical symptoms of the disease but also suffered from social discrimination and prejudice. ${ }^{[1]}$ This article expresses the negative impact of mental illness stigma on the stigmatized group through the study of previous literature. The purpose of this article is to improve the public's stereotypes and prejudices of people with mental illness, so as to provide a basis for researchers to identify effective de-stigmatization strategies.
\end{abstract}

\section{Introduction}

$\mathrm{F}$ or a long time, people with mental illness have been in a dilemma situation. On the one hand, this target population have to struggle with the psychological and physical symptoms of the disease. Mental disorders such as depression, anxiety, bipolar disorder, and schizophrenia can bring hallucinations, anxiety, mood swings and other issues to individuals ${ }^{[2]}$. On the other hand, the stigma comes from social misunderstandings and stereotypes of mental disorders has resulted in secondary trauma to patients with mental illness. This article will analyze and discuss the negative effects of stigma on people with mental illness by exploring the conceptual background and types of stigma ${ }^{[3]}$. The purpose of this article is to help the stigmatized groups and researchers to further understand the stigma of mental illness by exploring the definition of stigma and its impact on patients with mental illness, so as to provide the further foundation for de-stigmatization education and other antistigma measures.

\section{Mental Illness Stigma Influence}

Although the living environment of patients with mental illness has been improved with the popularization of education and the advancement of social patterns, the social norm they live in is still full of discrimination and misunderstanding ${ }^{[4]}$. This article will elaborate on the negative impact of stigma on patients with mental illness from the perspectives of housing, occupation, healthcare as well as self-confidence and self-esteem.

*Corresponding Author:

Minhang Li,

University of Sydney, Sydney, Haymarket 2007 NSW, Australia;

Email:minhangli_usyd@163.com 


\subsection{Influence in Housing}

It is proved that there is a negative correlation between socioeconomic status and mental illness. The low income and economic difficulties affected by the stigma would bring housing challenges. Due to the cross-effects caused by stigma, the housing conditions of patients with mental illness also face severe challenges. Studies have shown that most people with mental illness labels have the problems of insufficient housing and lack of safety guarantees ${ }^{[2]}$. Even if this population have an independent source of income to pay the rent, their only option is in low-income communities with lower housing standards and a higher risk of crime ${ }^{[2]}$. Although in these low-income communities, people with mental illness still do not have an advantage in housing competition compared with other potential tenants as some proportion of patients with severe mental illness lack social and coping skills, which makes it difficult to meet the demand for competitive independent housing ${ }^{[5]}$. According to Kowalchuk, more than half of people with mental illness are dissatisfied with their living conditions ${ }^{[9]}$. The continuity and security provided by a safe and stable house may bring about symptom improvement for individuals with severe mental illness and facilitate the reconstruction of interpersonal relationships.

\subsection{Influence in Employment}

People with mental health problems are disadvantaged in the field of employment. Baldwin and Marcus point out that people with mental illness suffer from unexplained high and significant levels of negative wage differences at work ${ }^{[6]}$. The reasons for this difference can be explained by stigma and discrimination. People with mental illness also face barriers in the job-hunting process. For example, gaps in resumes resulted from mental illness may lead to these job applicants to be eliminated in the first step ${ }^{[4]}$. Another serious challenge is that people suffering from mental illness not only experience stigma when applying for jobs but when they return to work. They may be in an unsafe situation that suffering differential treatment from colleagues including bullying, ridicule and demotion ${ }^{[4]}$. In addition, the impact of self-stigma on occupation is particularly reflected in work value and work connection. Studies have shown that the decline in self-esteem and self-efficacy resulted from self-shame can cause the "why try effect" that people with mental illness who internalize stigma believe that they are not worthy and do not have the ability to work independently ${ }^{[3]}$.

\subsection{Influence in the Medical System and Healthcare-seeking}

Research has identified that stigma leads to various problems in healthcare, which directly or indirectly affect the access and quality of medical care for patients with mental disorders. A study on the medical and health of mental illness shows that compared with people without illness, people with mental illness are less likely to benefit from the medical system. ${ }^{[7]}$. Druss et al. conclude that people with mental illness receive significant less medical service than those without a mental illness label. Specifically, due to the lack of professionalism of some health professionals and the inherent stereotypes of mental illness, individuals with mentally ill report that it is difficult to avoid discrimination and derogation in the process of treatment in the medical system ${ }^{[1]}$.

There is an incomprehensible phenomenon exists in the workplace of healthcare, which is that the healthcare field usually simplifies the mental health issues of employees and describes it as a cultural phenomenon ${ }^{[1]}$. Healthcare practitioners are not encouraged to seek help with mental health in the workplace. These unprofessional operations push the mentally ill patients out of the medical and health system, thus increasing the difficulty of treatment. In addition, self-stigma is an obstacle for people with mental illness to actively seek health treatment as stigma may be an intangible factor that undermines the participation of people with mental illness. Another challenge is that even if individuals with mental illness overcome the adverse effects of the initial labelling, the "why try effects" due to self-stigma during the treatment phase may contribute to doubts that the treatment will not bring real positive effects and thus terminate treatment ${ }^{[3]}$. Studies have shown that up to $20 \%$ of people may discontinue treatment during the treatment of mental illness ${ }^{[8]}$.

\subsection{Influence in Self-confidence and Self-esteem}

Although not all people with mental illness have completed the process of internalizing stigma, a considerable number of patients internalize negative stereotypes that therefore affect their self-confidence and self-esteem. Explaining why the stigma of mental illness can affect self-confidence and self-esteem requires understanding the "why try effect". "Why try effect" includes three processes, which are self-discrimination, systemic mediation of self-esteem and self-efficacy, and the achievement of life goals or negative orientation causes value loss ${ }^{[8]}$. When mentally ill patients experience the process of understanding the public's stereotypes about the stigmatized group, agree with these labels and complete their own ste- 
reotypes, the "why try effect" occurs ${ }^{[8]}$. The influence of the "why try effect" on individuals suffering from mental illness is the negative and discriminatory attitudes experienced in the process of an individual's internalization. Individuals may refuse to try beneficial behaviours that are conducive to recovery with the aims of avoiding the negative impact of the public on enhanced labelling. After an individual internalizes the public's label against the stigmatized group, it will lead to lower self-confidence and self-esteem because of shame ${ }^{[9]}$. Specifically, people with mental illness experience both the "why try" effect and the dual impact of public stigma and self-stigma, leading to loss of sense of value in life, the frustration of self-esteem, and lack of confidence and continuity to take action ${ }^{[9]}$. The results of low self-esteem and low self-confidence not only affect routine rehabilitation but brings numerous negative consequences to work and life.

\section{Conclusion}

On the basis of previous literature research, this article focuses on the negative effects of stigma on mental patients from the aspects of accommodation, occupation, healthcare system, self-confidence and self-esteem. As stigma is a complex phenomenon, it is necessary to expand research on public stigma, self-stigma, and the impact of stigma on mentally ill patients, especially more investment in medical area since this field is closely related to rehabilitation. One limitation is that this paper only discusses part of the negative effects of stigma on mental patients, which lacks effective strategies to manage the negative consequences of stigma on mental patients. Another obstacle that needs to be improved is that the article only has a limited selection of the negative effects of stigma, ignoring the mental illness stigma influence on their families. Therefore, it is necessary to conduct further empirical research to find out the best strategy to improve the negative effects of stigma to reduce the stigmatized attitudes and behaviors of mental patients. In addition, more research needs to be invested in the content of the relationship between stigma and self-efficacy and self-esteem.

\section{References}

[1] Corrigan, P. W., Druss, B. G., Perlick, D. A. The impact of mental illness stigma on seeking and participating in mental health care. Psychological Science in the Public Interest, 2014, 15(2): 37-70.

[2] Corrigan, P. W., Kleinlein, P. The impact of mental illness stigma, 2005.

[3] Corrigan, P. W., Larson, J. E., Ruesch, N. Self-stigma and the "why try" effect: impact on life goals and evidence-based practices. World psychiatry, 2009, 8(2): 75 .

[4] Corrigan, P. W., Powell, K. J., Rüsch, N. How does stigma affect work in people with serious mental illnesses? Psychiatric rehabilitation journal, 2009, 35(5): 381.

[5] Corrigan, P. W., Wassel, A. Understanding and influencing the stigma of mental illness. Journal of psychosocial nursing and mental health services, 2008, 46(1): 42-48.

[6] Corrigan, P. W., Shapiro, J. R. Measuring the impact of programs that challenge the public stigma of mental illness. Clinical psychology review, 2010, 30(8): 907-922.

[7] Druss, B. G., Rosenheck, R. A., Desai, M. M., Perlin, J. B. Quality of preventive medical care for patients with mental disorders. Medical care, 2002, 40(2): 129-136.

[8] Edlund, M. J., Wang, P. S., Berglund, P. A., Katz, S. J., Lin, E., Kessler, R. C. Dropping out of mental health treatment: Patterns and predictors among epidemiological survey respondents in the United States and Ontario. American Journal of Psychiatry, 2002, 159(5): 845-851.

[9] Kowalchuk, J. Housing, poverty and mental illness: Breaking the triangle of disadvantage. Social Policy Research Unit, 2004. 